THEORIA ET HISTORIA SCIENTIARUM, VOL. VIII, $\mathrm{N}^{\circ} 1$

Ed. Nicolaus Copernicus University 2008

Ray Paton

\title{
Field and Transformation in Relation to Biological Form
}

\begin{abstract}
Ideas about field and transformation are especially important in modem physics and have also made an impact of biological thinking in various ways. The purpose of this paper is to look at some integrative ideas in biology that access field and transformational thinking, particularly in relation to form. The paper begins with a brief consideration of some philosophical schools of thought that may and do influence thinking about form. This is followed by a consideration of relations between field and form in the work of Gurwitsch and Thompson. Finally, Thompson's transformational approach to comparative forms is considered with regard to interactions between biological forms and their environments.
\end{abstract}

\section{Introduction}

Our meddling intellect

Mis-shapes the beauteous forms of things: -

We murder to dissect

from William Wordsworth's poem The Thorn

This paper explores some integrative ideas that can be applied to thinking about biological form. In so doing it seeks to build on the discussion from previous papers (Paton, 1997, 2000) by reflecting on a number of conceptual relations between form, field and transformation. There are also a number of methodological and philosophical issues running through this paper related to the role(s) of diagrammatic representations, mathematical modelling and the playoff between precision and generality in relation to form. The approach to analogical thinking and concept displacement which underlies this paper is 
provided by the notion of paramorphic model and the role of iconic and mathematical source domains in theory development (e.g., Harre 1986).

The notions of field and transformation are common in certain areas of contemporary biology such as development (e.g., Webster \& Goodwin 1996). However, the present paper seeks to examine more general, and in many ways abstract issues. In order to achieve this, we look at selected aspects of the work of two biologists who wrote from the early decades of the twentieth century: Alexander Gurwitsch and D'Arcy Wentworth Thompson. The reason for selecting these two people relates to the relational and integrative nature of their thinking which has acted as an important source of insight and/or inspiration to subsequent research. This paper is not an historical critique or review of the limitations of their work, neither does it seek to consider more recent developments in geometric morphometries (e.g., Bookstein 1991). Rather it is an exploration of some integrative ideas and their displacement across knowledge domains.

\section{Biological Forms}

Biological form is more than shape, more than static configuration of components in a whole. There is a continual flux of matter through a living organism, while its form is maintained ... In living systems, irreversible processes play a constructive and indispensable role. (Capra 1996, p. 18)

By definition, a model is a representation of one thing in terms of something else. The representation of biological form in diagrams and even replica models is an abstraction. As the idea of biological form is examined more fully it becomes clear that certain philosophical systems have influenced thinking in this area. The quotation by Capra (above) indicates that form is more than a shape or static conformation. Biological forms are not fixed, and even within a short time period they may undergo many subtle changes. For example, changes to the wing conformation of a bird in flight are much more varied compared to an aircraft wing. In this paper we shall focus on comparative snapshots of form.

The philosophical schools of thought that may influence thinking about biological form are quite broad and often an individual person's viewpoint may encompass an overlapping of ideas. Consider a brief excursion to illustrate the point. A Pythagorean view could be summarised by Galileo's phrase: "The book of Nature is written in the language of mathematics" . Within these terms we note that there are several approaches which seek to treat biological form either numerically, geometrically or algorithmically. The search for pattern is itself 
reflected in a Pythagorean stance if one takes pattern to be a kind of mathematical abstraction. A Platonic approach assumes that there are eternal or ideal forms. Given that biological forms change within and between life cycles, ideals are more likely to be expressed in mathematical terms for example, with ideas like symmetry, invariance and transformation.

The Aristotelian perspective, which in the present discussion is best expressed in the essentialist view, may be linked with Platonism but emphasises that a form is described through the sharing of certain essential properties among instances. In some ways this remains an important view in a biological appreciation of form. Goethe's thinking was strongly essentialist and exhibited an idealistic (Platonic) view with regard to biological morphology in the notion of a morphotype (Lenoir 1987). He thought of biological form in terms of "patterns of relationships within an organised whole" (cited by Capra 1996, p. 21). In this respect his views resonate with current thinking notably in systems theory and relational biology. Structuralist thinking also falls within this category as it places an emphasis on whole, organismic ideas and transformation. The application of the concept of a "field' was displaced to numerous disciplines in the early decades of the twentieth century and maintained a structuralist approach since a field is distributed and structured in space and maybe also in time (see e.g., Piaget 1971). Process theory is also an anti-essentialist approach and places a great deal of emphasis on the relational aspects of biological systems.

The notion of form is an abstract idea related to a species of something rather than an individual instantiation. For example, the form of Diodon (see Figure 1) or of the chlorophyll molecule or of the femur is the representation of an interpretation of a collection of individual instances. In discussing biological form, and even in examining the notion of form itself, the influence of these various philosophical systems can be ignored but not escaped.

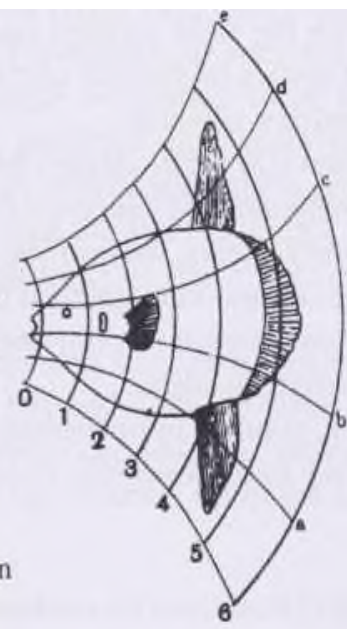




\section{Fields in Biology}

We begin our examination of fields in biology by looking at the modern origins of the field idea in physics. These are highly visual in nature and may be traced back in modern times to William Gilbert's work on magnetism De Magnete (1958). The orbis virtutis was Gilbert's original expression for what Faraday later called the field of force. In Faraday's terminology 'force' replaced Gilbert's 'virtue' (or 'power'). It is also worth noting that Gilbert's original concept did not involve the grounding of field dispositions in any substance, real or imaginary, in contrast with Maxwell's rotating tubes (see below), Faraday's elastic springs or even contemporary ideas based on energy distributions or exchange particles (Harre, 1998) ${ }^{2}$.

When Maxwell developed his theory of the electromagnetic field he thought of its behaviour in mechanical terms, as if it were a collection of wheels, pulleys and fluids. Miller (1996) notes that this visual metaphor formed the basis for the mathematical formulation of Maxwell's field equations and also how a more modern visualmechanical version deals with the behaviour of a field as if it is a distribution of harmonic oscillators. Maxwell's interpretation of Faraday was that the latter conceived of the field of space as being full of lines of force whose arrangement depended on that of bodies in the field and that mechanical (and electrical) action on each body was determined by the lines that abutted on it (Maxwell 1982). Following Hesse (1961) we note that a field in mathematical physics is usually described as a region of space where each point is characterised by some quantity or quantities that are functions of the space co-ordinates and of time. The nature of the quantities depends on the physical theory in which they occur. Although the field concept has its origins in magnetism we may also view it in terms of forces in general and for the present discussion in relation to what shapes the biology of form.

In order to appreciate relationships between form and field we now examine some ideas of Thompson and Gurwitsch. The validity and application of their work to contemporary views will not be examined critically but rather the use they made of the field idea. The material they produced was presented in a highly diagrammatic way and sought to combine a physicalist approach to biology with a number of mathematical ideas.

Beloussov (1997a) notes in his historical and biographical review paper about Gurwitsch's work on morphogenetic fields that:

${ }^{2}$ I am very grateful to Rom Harre for making this point very clear to me. 
...Gurwitsch introduces explicitly the idea of a 'field' as a supramolecular ordering principle governing the fate of cells; he referred to this principle or effect as a Kraftfeld or Geschehensfeld (field in which a force is exherted or in which Geschehen, events, oceur in an integrated, coordinated manner). (Beloussov 1997a, p. 773)

The notion of Kraftfeld clearly harks back to Gilbert's orbis virtutis. Closer reflection on this quotation also indicates the emergence of a coherent spatiotemporal ordering of a field of force or events that is integrated or 'glued' together. However, as Beloussov (1998) notes, Gurwitsch followed a Platonist tradition in focussing on "manifestations" ("proyavlenie" in Russian) of a field rather than the field itself". A coherent spatio-temporal ordering describes the continuity of a structure in space and time (Stromberg 1942) and from a developmental view can be related to the notion of a 'field of organisation' as discussed, for example, by Spemann (1938).

Gurwitsch's supramolecular ordering principle is grounded in a physical system. The field acts on molecules so that the spatial arrangements and molecular orderliness of the system is not derived solely from the discrete local interactions of the parts. Gurwitsch proposed that a cell creates a field around it by exploiting the energy released from exothermic reactions. This field contributes to transformations then taking place. There are many organisational scales in which coherence and transformational effects may operate in biosystems. A number of writers have examined some issues at the quantum level of organisation in relation to the ways biosystems exhibit multi-level information processing capabilities (e.g., Conrad 1996; Iganiberdiev 1999; Matsuno and Paton 2000).

Welch (1992) proposed an analogue field model of the metabolic state of a cell based on ideas from Quantum Field Theory. He reasoned that as the geometrical field concept of contemporary theoretical physics has reduced the material world to the intertwined web of dynamical space-time symmetries and invariance relations, so the structure of intracellular membranes and filaments, which are fractal in form, might generate or sustain local fields. Virtually all biomembranous structures in vivo can generate local electric fields and proton gradients. Enzymes can act as the energy transducing measuring devices of such local fields. In some ways we may say that the field provides a 'glue' which was not available at the individual, localised level of discrete components (see also, Paton 1997).

The role played by mechanical forces and stresses in organismal development has been around at least since Wilhelm His and within a contemporary setting can be seen in the notion of a field of mechanical stresses (e.g., Beloussov et al, 1997b). Thompson's view of living systems can be summed up in the following quote:

\footnotetext{
${ }^{3}$ Thanks to Lev Beloussov for his comments here.
} 
Living organism represents, or occupies, a field of force which is never simple, and which as a rule is of immense complexity (Thompson, 1942, p. 1030)

A statement that an organism or part is a representation of its environment presumes a great deal about interactions between system and surroundings. For example, how does a biological system 'measure' or 'interpret' its environment? This is particularly important when we come to examine the nature of models in relation to representation. In Thompson's sense a form is a representation. The representation made by an external observer of system-environment interactions is what is depicted in a model. In this case the diagrams that we see in books are at least representations of representations. So why is this important? Following the informal definition of model made earlier, we may say that a biological form models the environment. From Thompson's point of view, not only does an organism represent a field of force, the form is a kind of text (i.e., a diagram). He noted:

...the form of an object is a 'diagram of forces' (Thompson 1992, p. 11)

Here we are dealing with a mechanical object that is being represented in an engineering drawing. The diagram can be read. An external observer can interpret it. The points, lines and curves not only lead to a geometrical representation but also to a textual and transformational appreciation.

Diagrammatic reasoning and representations have played an important role in articulating relationships between form and field in physics (see e.g., Roche 1993). J. Z. Young (1978) applied a similar representational view to Thompson when he noted:

...the whole organism can be considered as a coded representation of its environment.

We can say that the wings of a bird 'represent' the air.. (Young 1978, p. 43)

An immediate question that arises from this quotation is the meaning(s) of "coded". The idea could be related to several issues such as:

1. the process of forming the wing may be a coded process or involve various codings or,

2. the interaction(s) between wing and air is coded or,

3. the interpretation(s) made by an external observer require a decoding activity.

With regard to the first case this must take account of genetic, epigenetic and developmental interactions within the organism and between the organism and its environment. The second case may be taken as related to an engineering or more properly an ecological engineering stance (not least in reliance on some teleonomic thinking). Also, the focus is on the complementarity between wing and air in the elaboration of an econiche. In the third case we make explicit reference to the interpretations of the observer-biologist. As we discuss later, the 
expression of comparative forms using co-ordinate transformations relies on an interpretative modelling framework.

If the wings of a bird "represent" the air then we are dealing with a modelling situation. This modelling situation has an ecological context. The air is an environmental factor. We briefly explore this ecological context using some Gibsonian ideas as interpreted by Harre (1987). The context of Harre's illustration is to explain complementarity and measurement in quantum mechanics. Harre notes how affordances can be viewed as dispositions of physical things relativised to that with which they interact. What a piece of apparatus measures is shaped by that apparatus. In the example of the preparation of liquid bromine the retort is shaped by the exigencies of condensing vapours and the existence of liquid bromine is made possible as a material stuff by that apparatus. There is a complementarity between the two things. Similarly, although it is not a device like a retort, a wing can be considered as an apparatus realising the affordance of the wing/air couple, namely, dispositions of the air only made available to wings (Harre 1998). ${ }^{4}$

Representation due to measurement (interaction) becomes an ecological idea. This is also seen in subtle representational comments made by Medawar on the relation between form and habitat on Thompson's transformational approach:

Orthagoriscus is a Diodon living in some quite remarkably non-Euclidean principality of the ocean, or that Diodon is an Orthagoriscus of ordinary Cartesian seas (Medawar 1982, p. 239)

In the case of Medawar as the external observer, the 'ocean' is non-Euclidean and the 'sea' Cartesian. However, neither the sea nor the ocean are mere mathematical constructs. In this sense 'sea' and 'ocean' are environments to be represented not only by an external observer but also by a biological system ${ }^{5}$. The interpretations and representations of form are also ecological.

The potential for displacement of concepts between text and form is large and diverse (Paton 1997). It has already been mentioned in the quote by Young (ibid.) that a link can be made between a form and a "coded representation". Indeed, a lot of work on fractal descriptions of biological forms can be related to a program (i.e., a code) that generates a form using a graph-rewriting grammar. A simple example would be in the production of tree structures using sequential recursive algorithms although in the biological case each natural branching process proceeds in parallel. In the biological context the nature of the 'code' or program is problematic. For example, equating code with genome conflates genotype and

\footnotetext{
4 Again, thanks to Rom Harre for his comments on this subject.

${ }^{5}$ It is important to note that the relation between system-environment discussed here presumes neither a selectionist nor instructivist stance.
} 
phenotype. Is a genome instructions or data or definitions or all of these things? Although the capacity of biosystems to 'read' instructions may be invariant, the rules and their symbolic manipulations for doing so are not.

There are limits to the approach to system (form) and representation being applied here. It is not as straightforward as a selectionist approach in which a population of biosystems generates forms and the environment selects those better suited to reproduce. In this case form may not simply be about a diagram of forces but also has something to do with the operation of naturally selected structures. Furthermore, no discussion is being made about the generation of form from the embryo to the adult. Rather we are looking at form as if it were in a dialogue with an environment. Such a dialogue is represented in the field.

\section{Transformation}

...despite the fact that they are 'analytically unwieldy'., Cartesian Transformations have been influential, and very likely they will continue to be so and stimulate new thoughts and methods in the future (Bonner, in Thompson 1992 p. 268)

Bonner's comment (above) captures a fascinating angle on Thompson's contribution. The transformational approach he introduced was unwieldy. It was also inaccurate. However, it provided a powerful analogical context for comparing forms. The figures that he produced, such as Figure 1, continue to be reproduced and continue to inspire further ideas and thoughts - almost like intellectual catalysts. Indeed, there is a breadth of influence of Thompson's work across a large number of scientific and engineering domains. Some recent successful developments applied statistical ideas such as least squares and repeated median to representations of form based on homologous points in dimensionless polygons such as skeletons (e.g., Seigel and Benson 1982). However, the generality of such methods is unclear.

Transformations are valuable intellectual tools for integrating ideas and experiences and diagrams are a powerful medium for achieving and communicating this. Mathematical transformations are highly pervasive and integrative devices. The transformational approach in geometry is particularly instructive with regard to invariances. For example, affine transformations preserve parallel lines and conformal transformations preserve angularity. With regard to biological form, transformations are associated with growth, metamorphosis and more abstractly with comparative morphology. The 'field of organisation' (e.g., Spemann 1938) provides the conditions in which the transformation from (say) blastula $\longrightarrow$ gastrula takes place.

The idea of a co-ordinate transformation was introduced by Thompson and an example is summarised diagrammatically in Figure 1. Recall the previous 
note that Thompson considered a biological form to be a diagram of forces. The pictorial juxtaposition of two forms such as Diodon and Orthagoriscus provided an 'analogical bridge' (see Seigel and Benson 1982) between biological forms. It has been noted how a form and an environment can be modelled as a couple (see the Medawar quote above - i.e., Medawar 1982, p. 239).

Thompson noted:

I have deformed its (Diodon's) vertical coordinates into a system of concentric circles and its horizontal coordinates into a system of curves. (Thompson 1942, p. 1063)

Clearly, Thompson approximates this conformal transformation in the language of 'systems of circles and curves'. Medawar rightly comments how Thompson grasped the transformation as a whole (i.e., including angle, length and ratio). This is a valuable contribution. A variety of extracted or 'dissected- out' components are kept together and transformed together. This contrasted with the alternative allometric approach which was a discretizing method that looked at correlations between particular properties such as mass, volume, heart rate, wing span and so forth. As Seigel and Benson (1982) noted, because the need for a reference system, studies of allometric growth and morphological change often hit difficulties in identifying and measuring the location and extent of relative shape deformations.

Thompsonian transformations are primarily about two dimensional objects and so from a mathematical point-of-view we are dealing with a transformation from $\mathrm{R}^{2}$ to $\mathrm{R}^{2}$. However, the biological arguments go much deeper than this, addressing issues concerned with the interpretation of an environment or a habitat. This indicates that there is more to the transformation of form than mappings on a plane. Such a mathematical approach is incomplete. Rosen (1967) commented that the transformational theory presented by Thompson was solely empirical and devoid of any clue about the mechanism underlying the deformation between organisms. Needham (1968) noted how Thompson had a tendency to treat the mathematical descriptions as explanations. For example, questions could be asked about what changes and what remains the same. How these could be assessed as to their biological (as opposed to mathematical) relevance requires that we deal with ecological meaning and interpretation.

A mathematical approach requires further qualifications - not when it comes to computing the transformations but rather in making the original representations. For example, Needham's (1934) focus was more on the matter rather than the form of life (i.e., chemistry rather than mathematics). He developed a view that there was a common biochemical "ground-plan" among organisms and that the representational abstraction of one organism could be transformed to another. Rosen (1992) used a genetic argument to describe the Thompsonian notion of 
transformation as the vehicle for moving phenotypic "data" from one species to another, different one. Within this view the transformation can be applied to determine (decide) the genotype of one species given the phenotypes of the two species and genotype of one from which the transformation is applied.

There are also problems regarding the precision of a transformation. In this sense the procedure is problematic. However, the value of the approach lies in its generality (at the expense of precision). Distinctive forms can be collected together within a common transformational framework. For example, rather than detailing precise 2D representations it may be appropriate to approximate certain forms and then make comparisons. A number of abstract mathematical ideas can be used to facilitate the displacement of ideas across these domains. A transformational approach as initiated by Thompson and developed for example by Needham, Medawar, Waddington and Rosen provides an important integrative construct for biological thinking namely, the relational properties of biological systems.

\section{Concluding Remarks - the Search for 'Glue'}

Fields are coherent structures and so glued together. In that it is a dynamically changing coherent structure, a biological form is within itself glued together. There is also a gluing or coupling between form and environment which constitutes an ecological complementarity between the two. These notions of form, field, and coherence presuppose both a closure and a dialogue (i.e., interaction). There are many ways to represent a form not just from the viewpoint of different mathematical and statistical systems but also with regard to ecological interpretation. The analogy of form as field can be seen in Thompson's statement that a form is a diagram of forces. The diagram as a whole represents a field. Some 'glues' are invariant when a transformation is made though others may not be. There are key conceptual relations between invariance, transformation and 'glue'. A mathematical metaphor may be used in a catechretic manner to enrich the 'glue' vocabulary. Specifically, we may apply the topological idea of a colimit operation to the adherence, combination and coherence of a pattern (Ehresmann \& Vanbremeersch 1987; Ehresmann 1997). This aspect of the work is the subject of ongoing dialogue and investigation.

Acknowledgements: I am grateful to Lev Beloussov, Andree Ehresmann and Koichiro Matsuno for a number of interesting and helpful discussions. I am especially grateful to Rom Harre for his detailed comments and valuable suggestions on an earlier draft of this paper. Thanks to Cambridge University Press for permission to reproduce Figure 1. 


\section{References}

Beloussov, L. V. (1997a). Life of Alexander G. Gurwitsch and his relevant contribution to the theory of morphogenetic fields. Int J Dev Biol 41, 6, 771-7.

Beloussov, L. V. (1997b). Morphogenetic Dynamics in Tissues: Expectations of Developmental and Cell Biologists, in Att, W., Deutsch, A. \& Dunn, G.(eds) Dynamics of Cell and Tissue Motion, Birkhauser Verlag, 215-219.

Beloussov, L. V. (1998). Personal communication.

Bookstein, F. L. (1991). Morphometric Tools for Landmark Data: Geometry and Biology. N.Y., Cambridge Univ. Press. An overview of the approach can be found at URL http://life.bio.sunysb.edu/morph/glossary/

Capra, F. (1996). The Web of Life. London: HarperCollins.

Conrad, M. (1996). Cross-scale Information Processing in Evolution, Development and Intelligence, BioSystems, 38, 97-109.

Ehresmann, A. C. \& Vanbremeersch, J-P. (1987). Hierarchical evolutive systems: mathematical model for complex systems. Bull. Math. Biol., 49, 1, 13-50.

Ehresmann, A. C (1997). Personal communication.

Gilbert, W. (1958). De Magnete translated and edited by P. Fleury Mottelay. New York, Dover.

Harre, R. (1986). Varieties of Realism: a Rationale for the Natural Sciences, Blackwell, Oxford. Harre, R., (1988), Parsing the Amplitudes, in Brown, H. R. \& Harre, R. (eds), Philosophical Foundations of Quantum Field Theory, Oxford: Clarendon Press, pp. 59-71.

Harre, R. (1998). Personal communication.

Hesse, M. (1961), Forces and Fields A Study of Action at a Distance in the History of Physics, London: Thomas Nelson and Sons.

Igamberdiev, A. U. (1999), Foundations of Metabolic Organisation: Coherence as a Basis of Computational Properties in Metabolic Networks, BioSystems, 50, 1-16.

Lenoir, T. (1987). Goethe's biological thought, in: Amrine, F, Zucker, F. J. \& Wheeler, H. (eds), Goethe and the Sciences: a Reappraisal, pp. 17-28. Dordrecht, D. Reidel.

Matsuno, K. \& Paton, R.C. (2000), Is there a Biology of Quantum Information, BioSystems.

Maxwell, J. C. (1982). A Dynamical Theory of the Electromagnetic Field, edited and introduced by T. F. Torrance. Edinburgh, Scottish Academic Press.

Medawar, P. B. (1947). Size, shape and age, in Le Gros Clark, W. E. \& Medawar, P. B. (eds), Essays on Growth and Form. Oxford, Clarendon Press.

Medawar, P. B. (1982). D'Arcy Thompson and Growth and Form, in: Pluto's Republic, Oxford University Press: Oxford, 228-241. This article was first published in 1958.

Miller, A. I. (1996). Insights of Genius, Imagery and Creativity in Science and Art. Copernicus: NY.

Needham, J. (1934). Chemical Heterogony and the Ground-Plan of Animal Growth, Biol. Rev., 9, 79-109. 
Needham, J. (1968). Biochemical Aspects of Form and Growth, in Whyte, L. L. (ed) op. cit., 77-90.

Paton., R. C. (1992). Towards a Metaphorical Biology. Biology and Philosophy 7, 279- -294.

Paton, R. C. (1H97). Glue, Verb and Text Metaphors in Biology. Acta Biotheoretica 45, 115.

Paton, R. C. (2000). Systemic Metaphor and Integrative Biology, Theoria et Historia Scientiarum, in press.

Piaget, J. (1991 ). Structuralism, RKP: London.

Roehe, J. J. (1993). The Semantics of Graphics in Mathematical Natural Philosophy, in Mazzolini, R. G. (ed), Non verbal Communication in Science Prior to 1900, Firenze: L. S. Olschki.

Rosen, R. (1967). Optimality in Biology, London: Butterworths.

Rosen, R. (1991). Life Itself, NY: Columbia University Press.

Seigel, A. F. \& Benson, R. H. (1982). A Robust Comparison of Shapes, Biometrics, 38, 341350.

Spemann, H. (1938). Embryonic Development and Induction, Yale University Press.

Strómberg, G. (1942). Coherence in the physical world, Phil, of Science, 9, 4, 323-334.

Thompson, D'Arcy W. (1942). On Growth and Form. A New Edition. University Press: Cambridge University Press. Also the 1992 Canto edition with Cambridge University Press.

Webster, G. \& Goodwin, B. C. (1996). Form and Transformation Generative and Relational Principles in Biology. Cambridge: CUP.

Welch, G. R. (1992). An analogical field construct in cellular biophysics: history and present status. Prog. Biophys. molec. Biol. 57: 71-128.

Whyte, L. L. (ed) (1968). Aspects of Form: a Symposium on Form in Nature and Art. Second Edition, London: Lund Humphries.

Young, J. Z. (1978). Programs of the Brain. Oxford: OUP. 